\title{
A RADICAL FOR RIGHT NEAR-RINGS: THE RIGHT JACOBSON RADICAL OF TYPE-0
}

\author{
RAVI SRINIVASA RAO AND K. SIVA PRASAD
}

Received 23 July 2005; Revised 19 July 2006; Accepted 25 July 2006

The notions of a right quasiregular element and right modular right ideal in a near-ring are initiated. Based on these $J_{0}^{r}(R)$, the right Jacobson radical of type- 0 of a near-ring $R$ is introduced. It is obtained that $J_{0}^{r}$ is a radical map and $N(R) \subseteq J_{0}^{r}(R)$, where $N(R)$ is the nil radical of a near-ring $R$. Some characterizations of $J_{0}^{r}(R)$ are given and its relation with some of the radicals is also discussed.

Copyright (c) 2006 Hindawi Publishing Corporation. All rights reserved.

\section{Introduction}

Throughout this paper, $R$ stands for a right near-ring. The structure of matrix near-rings was studied by Rao in $[3,4]$. It is clear from these papers that right Jacobson-type radicals have an important role to play in the study of Meldrum-van der Walt matrix near-rings. This motivated the authors to develop the right Jacobson-type radicals of near-rings. The aim of this paper is to give a good beginning in this direction of investigation. Left quasiregularity was introduced and studied in near-rings. In this paper, right quasiregularity is developed, and right modules of near-rings and the right Jacobson radical of type- 0 are studied.

In Section 2, the notions of a right quasiregular element and a right modular right ideal are introduced. Using these right 0 -primitive ideal, right 0 -primitive near-ring, and $J_{0}^{r}(R)$, the right Jacobson radical of $R$ of type- 0 are introduced. A nil subset of $R$ is right quasiregular and the constant part of $R$ is also right quasiregular. It is shown that $J_{0}^{r}$ is a radical map and $J_{0}^{r}(R)$ is the largest right quasiregular ideal of $R$. Moreover, $N(R)$, the nil radical of $R$, is contained in $J_{0}^{r}(R)$ and that $P(R) \subseteq N(R) \subseteq J_{0}^{r}(R)$. If $R$ is a zero-symmetric near-ring with DCC on the left $R$-subgroups of $R$, then $J_{0}(R) \subseteq J_{0}^{r}(R)$.

In Section 3, a right $R$-group of type-0 is introduced. It is shown that $G$ is a right $R$ group of type- 0 if and only if $G$ is right $R$-isomorphic to $R / K$, for some maximal right modular right ideal $K$ of $R$. A right 0 -primitive ideal of $R$ is a prime ideal of $R$. An ideal $P$ of a d.g. near-ring $R$ is a right 0 -primitive ideal of $R$ if and only if $P=(0: G)$, for some right $R$-group $G$ of type- 0 . 
In Section 4, the right Jacobson radical of type-0 of a biregular near-ring is studied. For such a near-ring $R$, it is shown that $J_{0}^{r}(R)=J_{0}(R)=\{0\}$. Moreover, if $R$ is biregular, then an ideal $P$ of $R$ is right 0 -primitive if and only if $P$ is left 0 -primitive if and only if $P$ is a maximal right and left modular ideal. It is shown by an example that in general $J_{0}^{r}(R)$ differs from the existing Jacobson-type radicals of $R$ and the $J_{0}^{r}$-radical class contains almost all the classes of near-rings with trivial multiplication.

\section{Right quasiregularity and the right $J_{0}$-radical}

Throughout this paper, $R$ is a right near-ring.

Definition 2.1. An element $a \in R$ is called right quasiregular if and only if the right ideal of $R$ generated by the set $\{x-a x \mid x \in R\}$ is $R$.

Remark 2.2. Note that unlike in the left quasiregularity case, if $R$ is a near-ring and $a \in R$, then the right ideal of $R$ generated by the set $\{x-a x \mid x \in R\}$ is $R$ if and only if $a$ is in the right ideal of $R$ generated by the set $\{x-a x \mid x \in R\}$.

Definition 2.3. A right ideal (left ideal, ideal, subset) $K$ of $R$ is called a right quasiregular right ideal (left ideal, ideal, subset) of $R$, if each element of $K$ is right quasiregular.

Lemma 2.4. A nilpotent element of $R$ is right quasiregular.

Proof. Let $a$ be a nilpotent element in $R$, say $a^{n}=0$ for some positive integer $n$. Let $K$ be the right ideal of $R$ generated by $x-a x, x \in R$. Observe that $x=x-a^{n} x=(x-a x)+$ $(a x-a(a x))+\left(a^{2} x-a\left(a^{2} x\right)\right)+\cdots+\left(a^{n-1} x-a\left(a^{n-1} x\right)\right) \in K$. Therefore, $K=R$ and hence $a$ is right quasiregular.

One of the major differences between right and left quasiregular elements of $R$ and hence between its right and left Jacobson-type radicals is the following.

Lemma 2.5. The constant part of $R$ is right quasiregular.

Proof. Let $R_{c}$ be the constant part of $R$ and $a \in R_{c}$. For $x \in R,(x+a)-a(x+a)=(x+$ $a)-a=x$. Therefore, $a$ is right quasiregular. So, $R_{c}$ is right quasiregular.

Lemma 2.6. Let e be a nonzero distributive idempotent in $R$. Then e is not a right quasiregular element of $R$.

Proof. Let $e$ be a nonzero distributive idempotent in $R$. Now $(0: e)=\{a \in R \mid e a=0\}$ is a right ideal of $R$. Also $x-e x \in(0: e)$ for all $x \in R$. But $e$ is not in $(0: e)$. So $(0: e) \neq R$. Therefore, $e$ is not right quasiregular.

Definition 2.7. A right ideal $K$ of $R$ is called right modular if there is an element $e \in R$ such that $x-e x \in K$ for all $x \in R$. In this case $K$ is said to be right modular by e.

Proposition 2.8. Let $e \in R$. Then e is right quasiregular if and only if no proper right ideal of $R$ is right modular by $e$.

The proof follows from the definitions.

Remark 2.9. Let $K$ be a right ideal of $R$. If $K$ is right modular by $e$, then $e \in K$ if and only if $K=R$. 
Proposition 2.10. Let $K$ be a proper right ideal of $R$. If $K$ is, right modular, then $K$ is contained in a maximal right ideal of $R$ which is also right modular.

The proof of this result is easy and hence omitted.

Proposition 2.11. Let $K$ and $L$ be right modular right ideals of $R$ and $R=K+L$. Then $K \cap L$ is also a right modular right ideal of $R$.

Proof. Suppose that $K$ is right modular by $e_{1}$ and $L$ is right modular by $e_{2}$. Let $e_{1}=b_{11}+$ $b_{12}$ and let $e_{2}=b_{21}+b_{22}$, where $b_{11}, b_{21} \in K$ and $b_{12}, b_{22} \in L$. Let $e=b_{21}+b_{12}$. Let $r \in$ $R ; r-e r=r-\left(b_{21}+b_{12}\right) r=r-b_{12} r-b_{21} r=\left(r-e_{1} r\right)+\left(b_{11} r-b_{21} r\right) \in K ; r-e r=r-$ $\left(b_{21}+b_{12}\right) r=r-b_{12} r-b_{21} r=\left(r-b_{12} r+b_{22} r-r\right)+\left(r-e_{2} r\right) \in L$. Therefore, $r-e r \in$ $K \cap L$, and hence $K \cap L$ is a right modular right ideal.

Proposition 2.12. If $K_{1}, K_{2}, \ldots, K_{n}$ are maximal right modular right ideals of $R$ such that $\bigcap_{i=1}^{n} K_{i}=\{0\}$, then $R$ has a left identity.

The proof follows from Proposition 2.11.

Definition 2.13. $J_{1 / 2}^{r}(R)$ is the intersection of all maximal right modular right ideals of $R$ and if $R$ has no maximal right modular right ideals, then $J_{1 / 2}^{r}(R)=R$.

THeOREM 2.14. $J_{1 / 2}^{r}(R)$ is the largest right quasiregular right ideal of $R$.

Proof. Let $q \in J_{1 / 2}^{r}(R)$. Suppose that $q$ is not right quasiregular. Let $K$ be the right ideal of $R$ generated by the set $\{x-q x \mid x \in R\}$. Now $q \notin K$. By Zorn's lemma, we get a right ideal $M$, which is maximal for the property that $K \subseteq M, q \notin M$. Now $M$ is a maximal right ideal of $R$. $M$ is right modular right ideal of $R$ as $x-q x \in M$, for all $x \in R$. As $q$ $\in J_{1 / 2}^{r}(R), q \in M$, a contradiction. Therefore each element of $J_{1 / 2}^{r}(R)$ is right quasiregular. We see now that each right quasiregular right ideal of $R$ is contained in $J_{1 / 2}^{r}(R)$. Let $K$ be a right quasiregular right ideal of $R$. We claim that $K \subseteq J_{1 / 2}^{r}(R)$. Suppose that $K$ is not contained in $J_{1 / 2}^{r}(R)$. We get a maximal right modular right ideal $M$ such that $R=M+K$. Suppose that $M$ is right modular by $e ; e=m+k, m \in M, k \in K ; x-k x=x-(-m+e) x=$ $x-(-m x+e x)=x-e x+m x \in M$. Since $M \neq R, k$ is not right quasiregular, which is a contradiction. Therefore, $K \subseteq J_{1 / 2}^{r}(R)$. Hence, $J_{1 / 2}^{r}(R)$ is the largest right quasiregular right ideal of $R$.

Remark 2.15. As expected, $J_{1 / 2}^{r}(R)$ is not an ideal of $R$. For this, consider the nonabelian group $G$ of order 6 . Then $M_{0}(G)$ is a simple near-ring. Since $G$ has only one nonzero maximal normal subgroup, $M_{0}(G)$ has only one nonzero maximal right ideal which is right modular by the identity element of $M_{0}(G)$. So, $J_{1 / 2}^{r}\left(M_{0}(G)\right)$ is not an ideal of $M_{0}(G)$.

Remark 2.16. Let $K$ be a right ideal of $R$. We show that there is a unique largest ideal of $R$ contained in $K$. Now $\{0\}$ is an ideal of $R$ and $\{0\} \subseteq K$. Let $I$ and $J$ be ideals of $R$ and $I \subseteq K, J \subseteq K$. Now $I+J$ is an ideal of $R$. Since $K$ is a subgroup of $(R,+), I+J \subseteq K$. Similarly, if $I_{1}, I_{2}, \ldots, I_{n}$ are ideals of $R$ and $I_{j} \subseteq K$, for all $1 \leq j \leq n$, then we get that $I_{1}+I_{2}+\cdots+I_{n} \subseteq K$. Let $\left\{J_{i} \mid i \in \Delta\right\}$ be the collection of all ideals $T$ of $R$ such that $T \subseteq K$. Let $J=\sum_{i \in \Delta} J_{i}$. Now $J$ is an ideal of $R$. It is clear that $J \subseteq K$ as any element $a \in J$ can be written as $a=a_{i_{1}}+a_{i_{2}}+\cdots+a_{i_{k}}, a_{i_{j}} \in J_{i_{j}}, i_{j} \in \Delta$. Obviously, $J$ is the largest ideal of $R$ contained in $K$. 
Definition 2.17. The largest ideal of $R$ contained in $J_{1 / 2}^{r}(R)$ is denoted by $J_{0}^{r}(R)$ and is called the right Jacobson radical of $R$ of type- 0 .

Theorem 2.18. $R \rightarrow J_{0}^{r}(R)$ is a radical map.

Proof. (1) First suppose that $R$ has no maximal right modular right ideal. Now $R=J_{0}^{r}(R)$ and that $R / J_{0}^{r}(R)=\{0\}$. So $J_{0}^{r}\left(R / J_{0}^{r}(R)\right)=\{0\}$. Suppose now that $R$ has a maximal right modular right ideal. Let $\left\{M_{\alpha} \mid \alpha \in \Delta\right\}$ be the collection of all maximal right modular right ideals of $R$. Since $M_{\alpha}$ is a maximal right modular right ideal of $R$ and $J_{0}^{r}(R) \subseteq M_{\alpha}$, $M_{\alpha} / J_{0}^{r}(R)$ is a maximal right modular right ideal of $R / J_{0}^{r}(R)$ for all $\alpha \in \Delta$. So $J_{0}^{r}\left(R / J_{0}^{r}(R)\right) \subseteq$ $\bigcap_{\alpha \in \Delta}\left(M_{\alpha} / J_{0}^{r}(R)\right)=\left(\bigcap_{\alpha \in \Delta} M_{\alpha}\right) / J_{0}^{r}(R)$. Since $J_{0}^{r}(R)$ is the largest ideal of $R$ contained in $\bigcap_{\alpha \in \Delta} M_{\alpha}$, we get that the largest ideal of $R / J_{0}^{r}(R)$ contained in $\bigcap_{\alpha \in \Delta}\left(M_{\alpha} / J_{0}^{r}(R)\right)$ is the zero ideal. Therefore, $J_{0}^{r}\left(R / J_{0}^{r}(R)\right)=\{0\}$.

(2) Let $h$ be a homomorphism of the near-ring $R$ onto a near-ring $S$. If $S$ has no maximal right modular right ideal, then $J_{0}^{r}(S)=S$. Then clearly $h\left(J_{0}^{r}(R)\right) \subseteq S=J_{0}^{r}(S)$. Suppose that $S$ has a maximal right modular right ideal. Let $\left\{N_{\alpha} \mid \alpha \in \Delta\right\}$ be the collection of all maximal right modular right ideals of $S$. Now $h^{-1}\left(N_{\alpha}\right)$ is a maximal right modular right ideal of $R$ for each $\alpha \in \Delta$. Let $M_{\alpha}=h^{-1}\left(N_{\alpha}\right), \alpha \in \Delta$. We have that $h\left(h^{-1}\left(N_{\alpha}\right)\right)=N_{\alpha}$, for all $\alpha \in \Delta$, and also $J_{0}^{r}(R) \subseteq \bigcap_{\alpha \in \Delta} M_{\alpha}$. So $h\left(J_{0}^{r}(R)\right) \subseteq h\left(\bigcap_{\alpha \in \Delta} M_{\alpha}\right) \subseteq \bigcap_{\alpha \in \Delta} h\left(M_{\alpha}\right)=\bigcap_{\alpha \in \Delta} N_{\alpha}$. Since $h\left(J_{0}^{r}(R)\right)$ is an ideal of $S$ and $J_{0}^{r}(S)$ is the largest ideal of $S$ contained in $\bigcap_{\alpha \in \Delta} N_{\alpha}$, $h\left(J_{0}^{r}(R)\right) \subseteq J_{0}^{r}(S)$. Therefore, $R \rightarrow J_{0}^{r}(R)$ is a radical map.

We denote the ideal of $R$ generated by an element $a$ of $R$ by (a). The following result is obvious in view of Theorem 2.14.

Theorem 2.19. $J_{0}^{r}(R)=\{a \in R \mid(a)$ is a right quasiregular ideal $\}$.

THeorem 2.20. $J_{0}^{r}(R)$ is the largest right quasiregular ideal of $R$.

The proof follows from Theorem 2.14.

Theorem 2.21. The nil radical $N(R)$ of $R$ is contained in $J_{0}^{r}(R)$.

The proof follows from Lemma 2.4 and Theorem 2.20.

CoRollary 2.22. $P(R) \subseteq N(R) \subseteq J_{0}^{r}(R)$, where $P(R)$ is the prime radical of $R$.

Proof. We know that $P(R) \subseteq N(R)$. Therefore from Theorem 2.21, $P(R) \subseteq N(R) \subseteq J_{0}^{r}(R)$.

THeORem 2.23. Let $R$ be a zero symmetric right near-ring with DCC on left $R$-subgroups of R. Then $J_{0}(R) \subseteq J_{0}^{r}(R)$.

Proof. By Pilz [2, Theorem 5.40], $J_{0}(R)$ is nilpotent. Therefore, $J_{0}(R) \subseteq N(R)$. By Theorem $2.21, N(R) \subseteq J_{0}^{r}(R)$. Hence, $J_{0}(R) \subseteq J_{0}^{r}(R)$.

Definition 2.24. The largest ideal contained in a maximal right modular right ideal of $R$ is called a right 0 -primitive ideal of $R$.

Remark 2.25. If $R$ is a ring, then $J_{0}^{r}(R)$ is the (right) Jacobson radical of $R$ and a right 0 -primitive ideal of the near-ring $R$ is a (right) primitive ideal of the ring $R$. 
THeOREm 2.26. If $J_{0}^{r}(R) \neq R$, then $J_{0}^{r}(R)$ is the intersection of all right 0 -primitive ideals of $R$.

Proof. $J_{0}^{r}(R)$ is an ideal contained in each maximal right modular right ideal of $R$. So $J_{0}^{r}(R)$ is contained in each right 0 -primitive ideal of $R$. Hence it is contained in the intersection of all right 0 -primitive ideals of $R$. On the other hand, the intersection of all right 0 primitive ideals of $R$ is an ideal contained in each maximal right modular right ideal of $R$ and that it is contained in $J_{0}^{r}(R)$.

Theorem 2.27. A maximal right modular ideal of a near-ring $R$ is a right 0 -primitive ideal of $R$.

Proof. Let $R$ be a near-ring and let $K$ be a maximal right modular ideal of $R$. Since $K$ is a proper right modular right ideal of $R, K$ is contained in a maximal right modular right ideal $M$ of $R$. Since $K$ is a maximal ideal of $R, K$ is the largest ideal contained in $M$. Hence, $K$ is a right 0 -primitive ideal of $R$.

Definition 2.28. $R$ is called a right 0 -primitive near-ring, if $\{0\}$ is a right 0 -primitive ideal of $R$.

Definition 2.29. A 0-primitive ideal of $R$ defined in Pilz [2] is called a left 0-primitive ideal of $R$ and similarly a left 0 -primitive near-ring.

Theorem 2.30. Let $P$ be an ideal of $R . P$ is a right 0 -primitive ideal of $R$ if and only if $R / P$ is a right 0 -primitive near-ring.

Proof. Let $P$ be a right 0 -primitive ideal of $R$. So we get a maximal right modular right ideal $M$ of $R$ such that $P$ is the largest ideal of $R$ contained in $M$. Now $M / P$ is a maximal right modular right ideal of $R / P$. Since $P$ is the largest ideal of $R$ contained in $M$, the zero ideal of $R / P$ is the largest ideal of $R / P$ contained in $M / P$. Therefore $R / P$ is a right 0 -primitive near-ring. Suppose now that $R / P$ is a right 0 -primitive near-ring. So we get a maximal right modular right ideal $M / P$ of $R / P$ such that the zero ideal of $R / P$ is the largest ideal of $R / P$ contained in $M / P$. Clearly $M$ is a maximal right modular right ideal of $R$. Since the zero ideal of $R / P$ is the largest ideal of $R / P$ contained in $M / P, P$ is the largest ideal of $R$ contained in $M$. Therefore, $P$ is a right 0 -primitive ideal of $R$.

THEOREM 2.31. A commutative right 0 -primitive near-ring is a field.

Proof. Let $R$ be a commutative right 0 -primitive near-ring. We get a modular maximal right ideal $M$ of $R$ such that $\{0\}$ is the largest ideal of $R$ contained in $M$. Suppose that $M$ is right modular by e. $x-e x \in M$, for all $x \in R$. Since $R$ is commutative, $M$ is an ideal of $R$. Therefore $M=\{0\}$. Since $x-e x \in M=\{0\}, x=e x=x e$. So $e$ is the identity element of $R$. Now $R$ is a commutative ring with identity. Since $M=\{0\}$ is a maximal ideal of $R$, $R$ is a field.

\section{Right $R$-groups of type- 0}

Definition 3.1. A group $(G,+)$ is called a right $R$-group if there is a mapping $(g, r) \rightarrow g r$ of $G \times R$ into $G$ such that $(1)(g+h) r=g r+h r,(2) g(r s)=(g r) s$, for all $g, h \in G$ and $r, s \in R$. 
A subgroup (normal subgroup) $H$ of a right $R$-group of $G$ is called an $R$-subgroup (ideal) of $G$, if $h r \in H$ for all $h \in H$ and $r \in R$.

$R$ is a right $R$-group. If $K$ is a subgroup of $(R,+)$ and $k r \in K$ for all $k \in K$ and $r \in R$, then $K$ is a right $R$-subgroup of $R$. Every right ideal of $R$ is an ideal of the right $R$-group $R$. Also, if $K$ is a right ideal of $R$, then $R / K$ is a right $R$-group, where $(x+K) r=x r+K$, for all $x+K \in R / K$ and $r \in R$.

Definition 3.2. Let $G$ be a right $R$-group. An element $g \in G$ is called a generator of $G$ if $g R=G$ and $g(r+s)=g r+g s$ for all $r, s \in R$. $G$ is said to be monogenic if $G$ has a generator.

Definition 3.3. Let $G$ and $H$ be right $R$-groups. A mapping $f: G \rightarrow H$ is called an $R$ homomorphism if $f(x+y)=f(x)+f(y)$ and $f(x r)=f(x) r$ for all $x, y \in G$ and for all $r \in R$. $G$ is said to be $R$-isomorphic to $H$ if there is a one-one $R$-homomorphism of $G$ onto $H$.

Proposition 3.4. Let $G$ be a right $R$-group. Then $G$ is monogenic if and only if there is a right modular right ideal $K$ of $R$ such that $G$ is $R$-isomorphic to $R / K$.

Proof. Let $G$ be a right $R$-group. Suppose that $G$ is monogenic. Let $g$ be a generator of $G$. Define $h: R \rightarrow G$ by $h(r)=g r$, for all $r \in R$. $h$ is an $R$-homomorphism of $R$ onto $G$. Let $K$ be the kernel of $h . K=\{r \in R \mid h(r)=0\}$ is a right ideal of $R$. Therefore $R / K$ is $R$-isomorphic to $G$. We get $b \in R$ such that $g=g b$. For each $x \in R, g x=g b x$. Now $g(x-b x)=0$, that is, $x-b x \in K$. So $K$ is modular by $b$. Conversely, suppose that $K$ is a right ideal of $R$ modular by $e$ and $R / K$ is $R$-isomorphic to $G$. Let $f$ be an $R$-isomorphism of $R / K$ onto $G$. Let $f(e+K)=g$. We see that $e+K$ is a generator of the right $R$-group $R / K$. Let $r, s \in R$. Now $r-e r \in K$. So $r+K=e r+K=(e+K) r \in(e+K) R$ and hence $(e+K) R=R / K$. Also $(r+s)-e(r+s), r-e r, s-e s \in K$. Let $k=r-e r$ and let $t=s-e s$. So $r=k+e r, s=t+e s$. Since $(r+s)-e(r+s) \in K$, we get that $(k+e r)+(t+e s)-e(r+$ $s)=k+(e r+t-e r)+e r+e s-e(r+s) \in K$ and that $e r+e s-e(r+s) \in K$. Therefore $e(r+s)+K=(e r+e s)+K$. So $(e+K)(r+s)=(e r+K)+(e s+K)=(e+K) r+(e+K) s$. This shows that $e+K$ is a generator of $R / K$. So $g$ is a generator of $G$ and hence $G$ is monogenic.

Proposition 3.5. Let $K$ be a right ideal of $R$. Then $K$ is right modular if and only if there is a right $R$-group $G$ with a generator $g$ such that $K=(0: g)$.

Proof. Suppose that $K$ is right modular by $e$. As seen in the above proposition $e+K$ is a generator of the right $R$-group $R / K$. Now $r \in(K: e+K) \Leftrightarrow e r+K=K \Leftrightarrow e r \in K \Leftrightarrow$ $r \in K$. Therefore $K=(K: e+K)$. Conversely suppose that $g$ is a generator of the right $R$-group $G$ and $(0: g)=K$. Since $g R=G$, we get $e \in R$ such that $g e=g$. Let $r \in R$. Now $g(r-e r)=g r-g r=0$. Therefore $r-e r \in(0: g)=K$. Hence, $K$ is right modular by $e$.

Definition 3.6. Let $G$ be a right $R$-group. $G$ is said to be simple if $G \neq\{0\}$ and $\{0\}$ and $G$ are the only ideals of $G$.

Definition 3.7. A monogenic right $R$-group $G$ is said to be a right $R$-group of type-0 if $G$ is simple. 
Proposition 3.8. Let $G$ be a right $R$-group. $G$ is a right $R$-group of type- 0 if and only if there is a maximal right modular right ideal $K$ of $R$ such that $G$ is $R$-isomorphic to $R / K$.

Proof. $G$ is a right $R$-group. Suppose that $G$ is of type- 0 . Let $g \in G$ be a generator. Therefore from the proof of Proposition 3.4, $G$ is $R$-isomorphic to $R / K$ for some right modular right ideal $K$ of $R$. Since $G$ is simple, we get that $R / K$ is also simple. Hence, $K$ is a maximal right ideal of $R$. Conversely, suppose that $G$ is $R$-isomorphic to $R / K$, where $K$ is a maximal right modular right ideal of $R$. Since $R / K \neq\{K\}$ has exactly two ideals, we get that $\{0\}$ and $G$ are the only ideals of $G$, where $\{0\} \neq G$. Let $K$ be right modular by $e$. So $e+K$ is a generator of $R / K$. Therefore, $G$ is also monogenic. Hence, $G$ is a right $R$-group of type- 0 .

Definition 3.9. Let $G$ be a right $R$-group. The annihilator of $G$ denoted by $(0: G)$ is defined as $(0: G)=\{a \in R \mid G a=\{0\}\}$.

If $A$ and $B$ are nonempty subsets of $R$, then $(A: B)$ denotes the set $\{r \in R \mid B r \subseteq A\}$.

Corollary 3.10. Let $K$ be a right modular right ideal of $R$. Then $(K: R) \subseteq K$.

Proof. Since $K$ is a right modular right ideal of $R$, by Proposition 3.5, there ia a right $R$ group $G$ with a generator $g$ such that $K=(0: g)$. Therefore, $K=(0: g) \supseteq(0: G)=(0$ : $R / K)=(K: R)$.

Proposition 3.11. Let $R$ be a zero-symmetric near-ring and let $K$ be a right ideal of $R$ right modular by e. Then $(K: R)=(K: e R)$ and the largest ideal of $R$ contained in $K$ is the largest ideal of $R$ contained in $(K: R)$.

Proof. Since $e R \subseteq R,(K: R) \subseteq(K: e R)$. Let $x \in(K: e R)$. Now eyx $\in K$, for all $y \in R$. But $y x-e y x \in K$, for all $y \in R$. Therefore, $y x \in K$, for all $y \in R$, that is, $x \in(K: R)$. So $(K: e R) \subseteq(K: R)$. Therefore, $(K: R)=(K: e R)$. Let $J$ be the largest ideal of $R$ contained in $K$. For $x \in J, R x \subseteq J \subseteq K$. Therefore, $J \subseteq(K: R)$. Let $I$ be an ideal of $R$ contained in $(K: R)$. By Corollary 3.10, $(K: R) \subseteq K$. So, $I \subseteq K$. Therefore, $I \subseteq J$. Hence, $J$ is the largest ideal of $R$ contained in $(K: R)$.

Proposition 3.12. Let $P$ be an ideal of a zero-symmetric near-ring $R$. $P$ is right 0 -primitive if and only if $P$ is the largest ideal of $R$ contained in $(0: G)$ for some right $R$-group $G$ of type-0.

Proof. Let $P$ be an ideal of a zero-symmetric near-ring $R$. Suppose that $P$ is a right 0 -primitive ideal of $R$. So we get a maximal right modular right ideal $K$ of $R$ such that $P$ is the largest ideal of $R$ contained in $K$. Now by Proposition 3.8, $R / K$ is a right $R$-group of type-0. By Proposition 3.11, $P$ is the largest ideal of $R$ contained in $(K: R)=(0: R / K)$. Conversely, suppose that $P$ is the largest ideal of $R$ contained in $(0: G)$, where $G$ is a right $R$-group of type- 0 . Now $G$ is $R$-isomorphic to $R / K$ for some maximal right modular right ideal $K$ of $R$. So $(0: G)=(0: R / K)=(K: R)$. Since $P$ is the largest ideal of $R$ contained in $(0: G)=(K: R)$, by Proposition 3.11, $P$ is the largest ideal of $R$ contained in $K$. Hence, $P$ is a right 0 -primitive ideal of $R$. 
Proposition 3.13. Let $G$ be a monogenic right $R$-group. If $R$ is a distributively generated (d.g.) near-ring then there is a subset $T$ of $G$ such that $h(a+b)=h a+h b$, for all $h \in T$ and $a, b \in R$, and $T$ generates $(G,+)$.

Proof. Let $G$ be a monogenic right $R$-group. Suppose that $R$ is a d.g. near-ring. Since $G$ is a monogenic right $R$-group, by Proposition 3.4, we get a right modular right ideal $K$ of $R$ such that $G$ is $R$-isomorphic to $R / K$. Let $f$ be a $R$-isomorphism of $G$ onto $R / K$. Let $S$ be the set of distributive elements of $R$, where $S$ generates $(R,+)$. It is clear that $\bar{S}=\{s+K \mid s \in S\}$ generates $(R / K,+)$. Let $s \in S$ and let $a, b \in R$. Since $s(a+b)=s a+s b$, we have that $s(a+b)+K=(s a+s b)+K$, that is, $(s+K)[a+b]=(s+K) a+(s+K) b$. Therefore, $T=\left\{f^{-1}(s+K) \mid s \in S\right\}$ is the required subset of $G$.

Proposition 3.14. Let $G$ be a monogenic right $R$-group. If $R$ is a d.g. near-ring, then $(0: G)$ is an ideal of $R$.

Proof. $G$ is a monogenic right $R$-group and $R$ is d.g. Let $S$ be the set of distributive elements of $R$. By Proposition 3.13, we get a subset $T$ of $G$ such that $h(x+y)=h x+h y$, for all $h \in T$ and $x, y \in R$, and $T$ generates $(G,+)$. Let $a, b \in(0: G), p, q \in R$, and let $g \in G$. $h(a+b)=h a+h b=0+0=0, h(-b)=-h b=0$ for all $h \in T$. Now $p=\delta_{1} s_{1}+\delta_{2} s_{2}+$ $\cdots+\delta_{n} s_{n}$, where $\delta_{i}= \pm 1$ and $s_{i} \in S$, for $1 \leq i \leq n$ :

$$
\begin{aligned}
p(q+a)-p q= & \left(\delta_{1} s_{1}+\delta_{2} s_{2}+\cdots+\delta_{n} s_{n}\right)(q+a)-\left(\delta_{1} s_{1}+\delta_{2} s_{2}+\cdots+\delta_{n} s_{n}\right) q \\
= & \delta_{1} s_{1}(q+a)+\delta_{2} s_{2}(q+a)+\cdots+\delta_{n} s_{n}(q+a) \\
& -\left(\delta_{1} s_{1} q+\delta_{2} s_{2} q+\cdots+\delta_{n} s_{n} q\right) \\
= & \delta_{1}\left(s_{1} q+s_{1} a\right)+\delta_{2}\left(s_{2} q+s_{2} a\right)+\cdots+\delta_{n}\left(s_{n} q+s_{n} a\right) \\
& -\delta_{n} s_{n} q-\delta_{n-1} s_{n-1} q-\cdots-\delta_{1} s_{1} q .
\end{aligned}
$$

Now

$$
\begin{aligned}
h[p(q+a)-p q]= & h\left(\delta_{1}\left(s_{1} q+s_{1} a\right)\right)+h\left(\delta_{2}\left(s_{2} q+s_{2} a\right)\right)+\cdots+h\left(\delta_{n}\left(s_{n} q+s_{n} a\right)\right) \\
& -h\left(\delta_{n} s_{n} q\right)-h\left(\delta_{n-1} s_{n-1} q\right)-\cdots-h\left(\delta_{1} s_{1} q\right) \\
= & \delta_{1}\left(h\left(s_{1} q\right)+\left(h s_{1}\right) a\right)+\delta_{2}\left(h\left(s_{2} q\right)+\left(h s_{2}\right) a\right)+\cdots+\delta_{n}\left(h\left(s_{n} q\right)\right. \\
& \left.+\left(h s_{n}\right) a\right)-\delta_{n} h\left(s_{n} q\right)-\delta_{n-1} h\left(s_{n-1} q\right)-\cdots-\delta_{1} h\left(s_{1} q\right) \\
= & \delta_{1} h\left(s_{1} q\right)+\delta_{2} h\left(s_{2} q\right)+\cdots+\delta_{n} h\left(s_{n} q\right)-\delta_{n} h\left(s_{n} q\right) \\
& -\delta_{n-1} h\left(s_{n-1} q\right)-\cdots-\delta_{1} h\left(s_{1} q\right)=0 .
\end{aligned}
$$

Also $h(a p)=(h a) p=0 p=0$. Since every element of $G$ is a finite sum of elements $h$, where $h \in T$ or $-h \in T$, we get that

(1) $g(a+b)=0$

(2) $g(-a)=0$; 
(3) $g(p+a-p)=0$;

(4) $g(p(q+a)-p q)=0$;

(5) $g(a p)=0$.

Therefore, $(0: G)$ is an ideal of $R$.

Corollary 3.15. Let $R$ be a d.g. near-ring and let $P$ be an ideal of $R$. Then $P$ is a right 0 -primitive ideal of $R$ if and only if $P=(0: G)$ for some right $R$-group $G$ of type- 0 .

Proof. Since a d.g. near-ring is zero-symmetric, the proof follows from Propositions 3.14 and 3.12.

Theorem 3.16. A right 0-primitive ideal of $R$ is a prime ideal of $R$.

Proof. Let $P$ be a right 0 -primitive ideal of $R$. We get a right $R$-module $G$ of type- 0 with a generator $g$ such that $P$ is the largest ideal of $R$ contained in $M=(0: g)=\{r \in R \mid g r=0\}$, $M$ is a maximal right modular right ideal of $R$. Let $A$ and $B$ be ideals of $R$ and $A B \subseteq P$. Suppose that $A \nsubseteq P$ and $B \nsubseteq P$. Since $A \nsubseteq P, g A \neq\{0\}$. Clearly $g A$ is a subgroup of $G$. Let $h \in G$ and let $a \in A$. $h=g r$, for some $r \in R$. $h+g a-h=g r+g a-g r=g(r+a-r) \in g A$, as $r+a-r \in A$. So $g A$ is a normal subgroup of $G$. Also $(g A) R=g(A R) \subseteq g A$. This shows that $g A$ is an ideal of $G$. Since $g A \neq\{0\}$ and $G$ is a right $R$-group of type- $0, g A=G$. Similarly, for $B$, also $g B=G$. Now $G \supseteq g A B=(g A) B=G B \supseteq g B=G$. Therefore, $g A B=$ $G$, a contradiction to the fact that $g A B=\{0\}$. So either $A \subseteq P$ or $B \subseteq P$. Hence, $P$ is a prime ideal of $R$.

\section{The right $\mathrm{J}_{0}$-radical of a biregular near-ring}

In this section, it will be shown that the right and left Jacobson radicals of type- 0 of a biregular near-ring $R$ are equal, and an ideal $P$ of $R$ is right 0 -primitive if and only if $P$ is left 0 -primitive.

We recall the following definition of Betsch (Pilz [2, Remark 3.49]).

Definition 4.1. A near-ring $R$ is called biregular if there exists a set $E$ of central idempotents of $R$ such that

(1) Re is an ideal of $R$ for all $e \in E$;

(2) for each $r \in R$, there exists an $e \in E$ such that $\operatorname{Re}=(r)$;

(3) $e+f=f+e$, for all $e, f \in E$;

(4) $e f, e+f-e f \in E$, if $e, f \in E$.

A biregular near-ring is zero-symmetric. Let $R$ be a biregular near-ring. Now $\{0\}=$ $(0)=\operatorname{Re}$, for some central idempotent $e$ of $R$. Also $e=e e \in \operatorname{Re}=\{0\}$. So $e=0$ and hence $R 0=\{0\}$. Therefore $R$ is zero-symmetric.

Theorem 4.2. Let $R$ be a biregular near-ring. Then $J_{0}^{r}(R)=\{0\}$.

Proof. Let $R$ be a biregular near-ring. Let $x \in J_{0}^{r}(R)$. Let $(x)$ be the ideal of $R$ generated by $x$. Now $(x)=(e)$, for some central idempotent $e \in R$. Therefore $e \in J_{0}^{r}(R) \subseteq J_{1 / 2}^{r}(R)$. Since $e$ is central, $e$ is a distributive idempotent. By Lemma 2.6, $J_{1 / 2}^{r}(R)$ contains no nonzero distributive idempotent. Therefore, $e=0$. Hence, $J_{0}^{r}(R)=\{0\}$. 
Theorem 4.3. Let $R$ be a biregular near-ring. Then $R$ is right (left) 0 -primitive if and only if $R$ is a nonzero simple near-ring with identity.

Proof. Let $R$ be a biregular near-ring. Suppose that $R$ is a right (left) 0 -primitive near-ring. Let $0 \neq a \in R$. Now $(a)=\operatorname{Re}$, for some central idempotent $e \neq 0$ in $R$. Since $e$ is a central idempotent, $(0: e)$ is an ideal of $R$. Now $R=\operatorname{Re}+(0: e)$ is a direct sum of the ideals $\operatorname{Re}$ and $(0: e)$. Since $R$ is right (left) 0 -primitive, $\{0\}$ is a prime ideal of $R$ by Theorem 3.16 (Pilz [2, Theorem 4.34]). Since $R$ is zero-symmetric, $\operatorname{Re}(0: e) \subseteq \operatorname{Re} \cap(0: e)=\{0\}$. So either $\operatorname{Re}=\{0\}$ or $(0: e)=\{0\}$. Since $e \neq 0, \operatorname{Re} \neq\{0\}$. Therefore, $(0: e)=\{0\}$. Hence, $R=\operatorname{Re}=$ (a). So $R$ is a simple near-ring with identity $e$. Conversely, suppose that $R$ is a nonzero simple near-ring with identity. Since $R$ is a near-ring with identity, $R$ has a maximal right (left) ideal $K$ and is right (left) modular by the identity. Since $R$ is simple, $\{0\}$ is the largest ideal of $R$ contained in $K$. Therefore, $\{0\}$ is a right (left) 0 -primitive ideal of $R$ and hence $R$ is right (left) 0-primitive.

Conollary 4.4. A right (left) 0 -primitive ideal of a biregular near-ring is a maximal ideal which is both right and left modular.

Proof. Let $R$ be a biregular near-ring and let $P$ be a right (left) 0 -primitive ideal of $R$. By Theorem 2.30, $R / P$ is a right (left) 0 -primitive near-ring. Since $R / P$ is also biregular, by Theorem $4.3, R / P$ is a simple near-ring with identity. So, $P$ is a maximal right and left modular ideal.

Corollary 4.5. Let $R$ be a biregular near-ring and let $P$ be an ideal of $R$. Then the following statements are equivalent:

(1) $P$ is a right 0 -primitive ideal of $R$;

(2) $P$ is a left 0 -primitive ideal of $R$;

(3) $P$ is a maximal ideal of $R$ which is both right and left modular.

Proof. By Corollary 4.4, (1) implies (3) and (2) implies (3). Let $P$ be a maximal right and left modular ideal of $R$. Since $P$ is a maximal right (left) modular ideal of $R, R / P$ is a simple near-ring with a left (right) identity. So $R / P$ is a simple near-ring with identity. Since $R / P$ is biregular, by Theorems 4.3 and 2.30, we get that (3) implies (1) and (3) implies (2).

Corollary 4.6. Let $(G,+)$ be a group with more than one element. Then $M_{0}(G)$ is a right and left 0 -primitive near-ring.

Proof. Since $M_{0}(G)$ is a nonzero simple biregular near-ring, it is a right and left 0primitive by Theorem 4.3.

Now we observe an interesting fact that unlike the left Jacobson-type radical classes, the $J_{0}^{r}$-radical class contains almost all the classes of near-rings with trivial multiplication.

We consider $J_{0}^{r}$-radical of a near-ring with trivial multiplication. Let $(R,+)$ be a group containing more than one element. Let $S$ be a nonempty subset of $R$ not containing 0 . The trivial multiplication on $(R,+)$ determined by $S$ is given by $a \cdot b=a$, if $b \in S$ and 0 , if $b \in S^{c}$, where $S^{c}$ is the complement of $S$ in $R$. Then $(R,+, \cdot)$ is a near-ring. 
Example 4.7. Let $R, S$, and $S^{c}$ be as defined above. Then $S^{c}$ is nilpotent and hence it is right quasiregular. Moreover, $R$ is right quasiregular if and only if $S^{c}$ is not a normal subgroup of $(R,+)$ of index 2 .

Let $a \in R$. Let $A=\{x-a x \mid x \in R\}$, let $B=\{x-a x \mid x \in S\}$, and let $C=\{x-a x \mid$ $\left.x \in S^{c}\right\}$. Now $A=B \cup C, B=\{x-a \mid x \in S\}=S+(-a)$, and $C=\left\{x \mid x \in S^{c}\right\}=S^{c}$. So $A=[S+(-a)] \cup S^{c}$. Let $K$ be the normal subgroup of $(R,+)$ generated by $A$. Clearly $K$ is a right ideal of $R$. Hence, $K$ is the right ideal of $R$ generated by $A$.

If $a, b \in S^{c}$, then $a b=0$. Therefore, $S^{c}$ is nilpotent and right quasiregular. Now suppose that $a \in S$. First assume that $S^{c}$ is not a normal subgroup of $(R,+)$. Since $R=S \cup S^{c}$, the normal subgroup of $(R,+)$ generated by $S^{c}$ contains an element $s \in S$. So $s \in K$. Since $s, s-a \in K, a=-(s-a)+s \in K$. Therefore, $a$ is right quasiregular.

Assume now that $S^{c}$ is a normal subgroup of $(R,+)$. Since $a \in S$, a is not in $S^{c}$. So $-a$ is not an element of $S^{c}$, as $S^{c}$ is a subgroup. Since $R=S^{c} \cup S, R=\left[S^{c}+(-a)\right] \cup[S+(-a)]$. Now $S^{c}$ and $S^{c}+(-a)$ are two right cosets of $S^{c}$ in $(R,+)$. Let $S^{c}, S^{c}+(-a), S^{c}+r_{\alpha}$, and $\alpha \in \triangle$ be the distinct right cosets of $S^{c}$ in $(R,+)$. So $R=S^{c} \cup\left[S^{c}+(-a)\right] \cup\left[\cup S^{c}+r_{\alpha}\right]$. The complement of $S^{c}+(-a)$ in $R$ is $S+(-a)=S^{c} \cup\left[\cup S^{c}+r_{\alpha}\right]$. If the index of $S^{c}$ in $(R,+)$ is 2 , then $S+(-a)=S^{c}$ and hence $A=S^{c}=K \neq R$. Thus $a$ is not right quasiregular.

Now assume that the index of $S^{c}$ in $(R,+)$ is not 2 . So, $S+(-a) \neq S^{c}$. We will show that $S+(-a)$ is not a normal subgroup of $(R,+)$. Suppose that $S+(-a)$ is a normal subgroup of $(R,+)$. Since $S^{c} \subseteq S+(-a), R=\left[S^{c}+(-a)\right] \cup[S+(-a)]$, and $\left[S^{c}+(-a)\right] \cap[S+(-a)]$ is empty, $S^{c}+(-a)=S+(-a)+(-a)$. Since $S^{c}$ is a proper subset of $S+(-a), S^{c}+(-a)$ is a proper subset of $S+(-a)+(-a)=S^{c}+(-a)$, a contradiction. Therefore, $S+(-a)$ is not a normal subgroup of $(R,+)$. So, the normal subgroup of $(R,+)$ generated by $S+(-a)$ contains an element $x+(-a)$ of $S^{c}+(-a), x \in S^{c}$. Now $x+(-a), x \in K$, and $a=-(x+$ $(-a))+x \in K$. Therefore, $a$ is right quasiregular.

Remark 4.8. Let $R, S$, and $S^{c}$ be as defined above and let $S^{c}$ be a normal subgroup of $(R,+)$ of index 2. Then, $S^{c}$ is a nilpotent ideal of $R, S^{c}=P(R)=J_{0}^{r}(R)=J_{2}(R)$. Moreover, $S^{c}$ is the set of right (left) quasiregular elements of $R$.

We see now by an example that there is a near-ring $R$ which has a left 0 -primitive ideal, but has no right 0 -primitive ideals and that the right and left Jacobson radicals of $R$ of type- 0 are different. We know that every prime ideal of a zero-symmetric near-ring $R$ with DCC on left $R$-subgroups of $R$ is left 0 -primitive, but there is a finite zero-symmetric near-ring $R \neq\{0\}$ in which no (proper) prime ideal is right 0 -primitive. Even though for a zero-symmetric near-ring $R$ with DCC on left $R$-subgroups of $R$, every left quasiregular left $R$-subgroup of $R$ is nilpotent, we see that there is a finite zero-symmetric right nearring $R$ in which no nonzero right quasiregular right $R$-subgroup of $R$ is nil.

Example 4.9. Let $(R,+)$ be a group containing more than two elements. Define a trivial multiplication in $R$ by $r s=r$ if $s \neq 0$ and 0 if $s=0$ for all $r, s \in R$. $R$ is a zero-symmetric right near-ring. Clearly, $R$ is a left $R$-group of type-2. Moreover, $R$ is simple. Therefore, $R$ is 2-primitive on the left $R$-group $R$, as $R R \neq\{0\}$. So $J_{2}(R)=\{0\}=J_{0}(R)$. But each element of $R$ is right quasiregular. Therefore, $J_{0}^{r}(R)=R$. If $R$ is finite, then obviously $R$ has DCC on right (left) $R$-groups of $R$, but no nonzero right quasiregular right ideal of $R$ is nilpotent. Moreover, the zero ideal of $R$ is prime but not right 0 -primitive. 
We recall some of the definitions and results of [3] which are required to observe that right Jacobson radicals are relevant for the study of near-rings in terms of matrix nearrings.

Matrix near-rings were introduced in [1].

Definition 4.10. Let $R$ be a zero-symmetric near-ring with identity. A subset $\left\{e_{i j} \mid 1 \leq\right.$ $i, j \leq n$ \} of distributive elements in $R$ is said to be a set of matrix units in $R$ if and only if $e_{11}+e_{22}+\cdots+e_{n n}=1$ and $e_{r s} e_{p q}=\delta_{s p} e_{r q}$, where

$$
\delta_{s p}= \begin{cases}1 & \text { if } s=p \\ 0 & \text { if } s \neq p\end{cases}
$$

Proposition 4.11. Let $R$ be a zero-symmetric near-ring with identity. $R=K_{1} \oplus K_{2} \oplus \cdots \oplus$ $K_{n}$, a direct sum of n pairwise isomorphic right ideals $K_{i}$ of $R$ as right $R$-groups if and only if $R$ has a set of matrix units $\left\{e_{i j} \mid 1 \leq i, j \leq n\right\}$. In this case $K_{i}=e_{i i} R$, for all $1 \leq i \leq n$.

As stated soon after [3, Corollary 15], we have the following.

Theorem 4.12. Let $R$ be a simple and d.g. near-ring with identity. Then $R$ is isomorphic to a matrix near-ring $M_{n}(S)$ if and only if $R$ has a set of matrix units $\left\{e_{i j} \mid 1 \leq i, j \leq n\right\}$.

Theorem 4.13. Let $R$ be a simple d.g. near-ring with DCC on right ideals of $R$ and $J_{1 / 2}^{r}=$ $\{0\}$. Suppose that any two minimal right ideals of $R$ are isomorphic as right $R$-groups. Then, $R=K_{1} \oplus K_{2} \oplus \cdots \oplus K_{n}$, a direct sum of minimal right ideals $K_{i}$ and is (isomorphic to) a matrix near-ring $M_{n}(S)$.

Proof. Since $J_{1 / 2}^{r}(R)=\{0\}$ and $R$ has DCC on right ideals of $R$, we get that the intersection of a finite number of maximal right modular right ideals of $R$ is zero. So, $R$ is a direct sum of a finite number of minimal right ideals $K_{1}, K_{2}, \ldots, K_{n}$ of $R$. By Proposition 2.12, $R$ has a left identity as the intersection of a finite number of maximal right modular right ideals of $R$ is zero. Since $R$ is a simple near-ring with left identity, it has an identity. Also, since by our assumption any two minimal right ideals of $R$ are isomorphic as right $R$ groups, by Proposition 4.11, $R$ has a set of matrix units $\left\{e_{i j} \mid 1 \leq i, j \leq n\right\}$. Therefore, by Theorem 4.12, $R$ is (isomorphic to) a matrix near-ring $M_{n}(S)$.

Example 4.14. We give an example of a nonring which satisfies the hypothesis of Theorem 4.13. Let $G$ be a finite simple nonabelian additive group. By [3, Corollary 19], $E\left(G^{2}\right)$ is isomorphic to the matrix near-ring $M_{2}(E(G))$. As mentioned soon after [3, Corollary 19], $E\left(G^{2}\right)=M_{0}\left(G^{2}\right)$. So, $M_{0}\left(G^{2}\right)$ is a simple d.g. near-ring with DCC on right ideals. Let $i \in\{1,2\}$. Let $G_{1}=G \times\{0\}$ and let $G_{2}=\{0\} \times G$. Since $G_{i}$ is a maximal (minimal) normal subgroups of $G^{2}, K_{i}=\left(G_{i}: G^{2}\right)=\left\{m \in M_{0}\left(G^{2}\right) \mid m(a) \in G_{i}\right.$, for all $\left.a \in G^{2}\right\}$ is a maximal right ideal of $M_{0}\left(G^{2}\right)$. Moreover, $K_{1} \cap K_{2}=\{0\}$. Thus $J_{1 / 2}^{r}\left(M_{0}\left(G^{2}\right)\right)=\{0\}$. This shows that $M_{0}\left(G^{2}\right)=K_{1} \oplus K_{2}$, where $K_{i}$ is a minimal right ideal of $M_{0}\left(G^{2}\right)$. Define $e_{i}: G^{2} \rightarrow G_{i}$ by $e_{i}\left(\left(a_{1}, a_{2}\right)\right)=\left(b_{1}, b_{2}\right)$, where $b_{j}=a_{i}$ if $j=i$ and 0 if $j \neq i$. Now $e_{i}$ is a group homomorphism and hence it is a distributive idempotent in $M_{0}\left(G^{2}\right)$ and $e_{i} M_{0}\left(G^{2}\right) \subseteq K_{i}$. Since $e_{1}$ and $e_{2}$ are orthogonal distributive idempotents in $M_{0}\left(G^{2}\right)$ and $e_{1}+e_{2}=1$, by [3, Proposition 2], we get that $e_{i} M_{0}\left(G^{2}\right)$ is a right ideal of $M_{0}\left(G^{2}\right)$. Thus, $K_{i}=e_{i} M_{0}\left(G^{2}\right)$. 
The mapping $e_{12}: G^{2} \rightarrow G^{2}$ defined by $e_{12}\left(\left(a_{1}, a_{2}\right)\right)=\left(a_{2}, 0\right)$ is an endomorphism of $G^{2}$. So, $e_{12}$ is a distributive element in $M_{0}\left(G^{2}\right)$. It is an easy verification that the mapping $h: e_{2} M_{0}\left(G^{2}\right) \rightarrow e_{1} M_{0}\left(G^{2}\right)$ defined by $h\left(e_{2} m\right)=e_{12}\left(e_{2} m\right)$ is an isomorphism of the right $M_{0}\left(G^{2}\right)$-groups. So, $K_{1}$ and $K_{2}$ are isomorphic as right $M_{0}\left(G^{2}\right)$-groups. Since a minimal right ideal $K$ of $M_{0}\left(G^{2}\right)$ is isomorphic to $K_{j}$ for some $j \in\{1,2\}$ as right $M_{0}\left(G^{2}\right)$-groups, we get that any two minimal right ideals of $M_{0}\left(G^{2}\right)$ are isomorphic as right $M_{0}\left(G^{2}\right)$ groups. So, $M_{0}\left(G^{2}\right)$ satisfies the hypothesis of Theorem 4.13 .

Example 4.15. Let $G$ be a finite simple nonabelian additive group. Now by Pilz [2, Corollary 7.48], $E(G)=M_{0}(G)$. So, $M_{0}(G)$ is a finite simple d.g. near-ring with identity. Moreover, $J_{2}\left(M_{0}(G)\right)=\{0\}$ and each minimal left ideal of $M_{0}(G)$ is isomorphic to $G$ as left $M_{0}(G)$-groups. Since each distributive element of $M_{0}(G)$ is an endomorphism of $(G,+), 0$ and the automorphisms of $(G,+)$ are the only distributive elements of $M_{0}(G)$. Therefore, $M_{0}(G)$ has no nontrivial matrix units. Hence, $M_{0}(G)$ is not isomorphic to a matrix nearring $M_{n}(S)$, where $n>1$.

\section{Acknowledgments}

The first author would like to thank the management of the Siddhartha Academy of General and Technical Education, Vijayawada for providing necessary facilities. This work is done under the U.G.C. Research Project no.MRP-336/04. The authors thank the referees for their valuable suggestions.

\section{References}

[1] J. D. P. Meldrum and A. P. J. van der Walt, Matrix near-rings, Archiv der Mathematik 47 (1986), no. 4, 312-319.

[2] G. Pilz, Near-Rings, rev. ed., North-Holland Mathematics Studies, vol. 23, North-Holland, Amsterdam, 1983.

[3] R. S. Rao, On near-rings with matrix units, Quaestiones Mathematicae 17 (1994), no. 3, 321332.

[4] Wedderburn-Artin theorem analogue for near-rings, Southeast Asian Bulletin of Mathematics 27 (2004), no. 5, 915-922.

Ravi Srinivasa Rao: Department of Mathematics, PG Centre, PB Siddhartha College of Arts and Sciences, Vijayawada 520010, Andhra Pradesh, India

E-mail address:dr_rsrao@yahoo.com

K. Siva Prasad: PG Department of Mathematics, JKC College, Guntur 522006, Andhra Pradesh, India E-mail address: siva235prasad@yahoo.co.in 


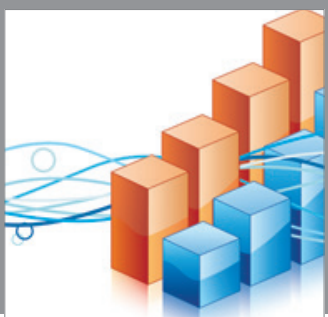

Advances in

Operations Research

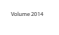

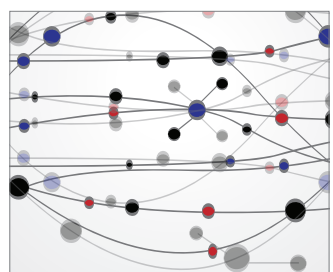

\section{The Scientific} World Journal
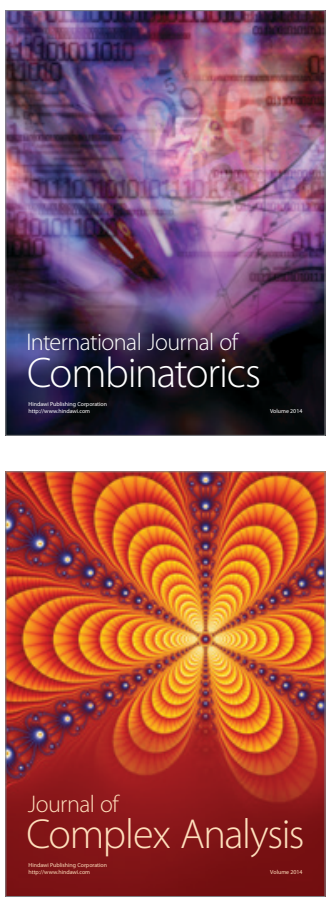

International Journal of

Mathematics and

Mathematical

Sciences
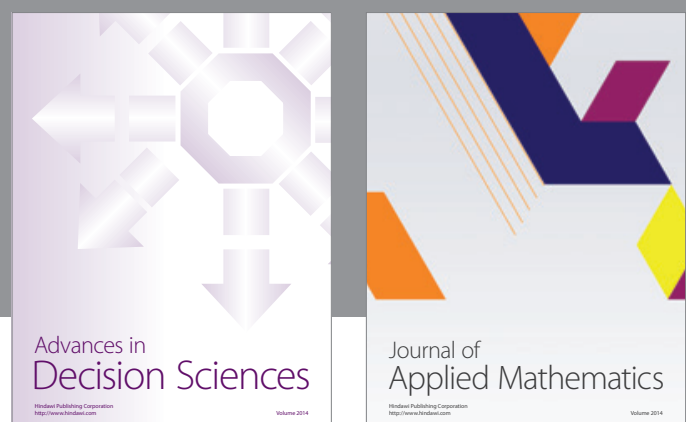

Journal of

Applied Mathematics
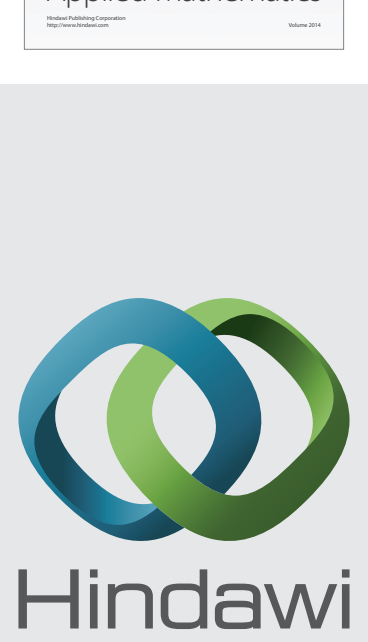

Submit your manuscripts at http://www.hindawi.com
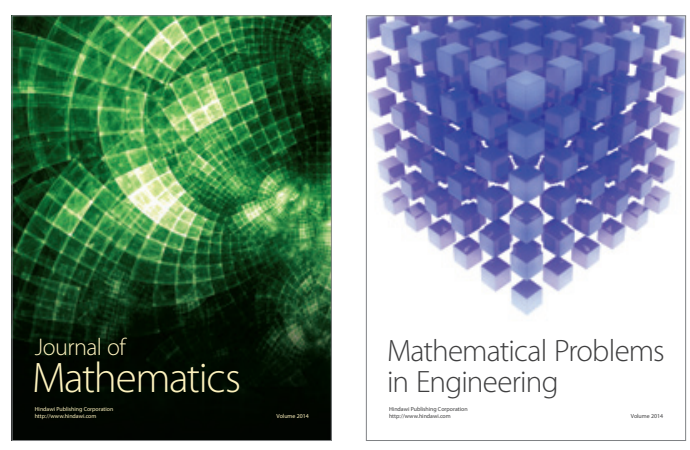

Mathematical Problems in Engineering
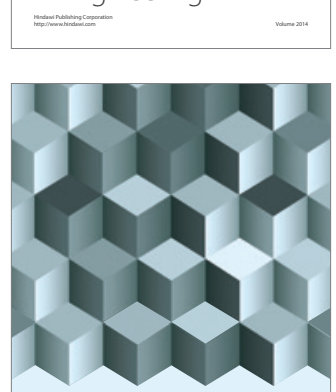

Journal of

Function Spaces
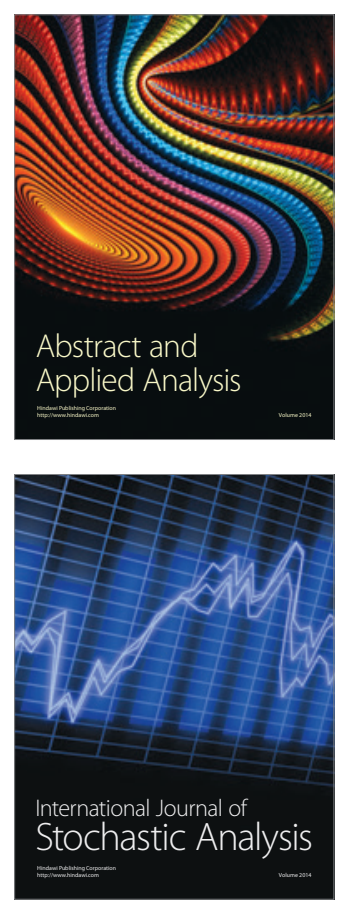

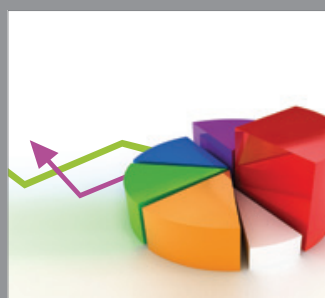

ournal of

Probability and Statistics

Promensencen
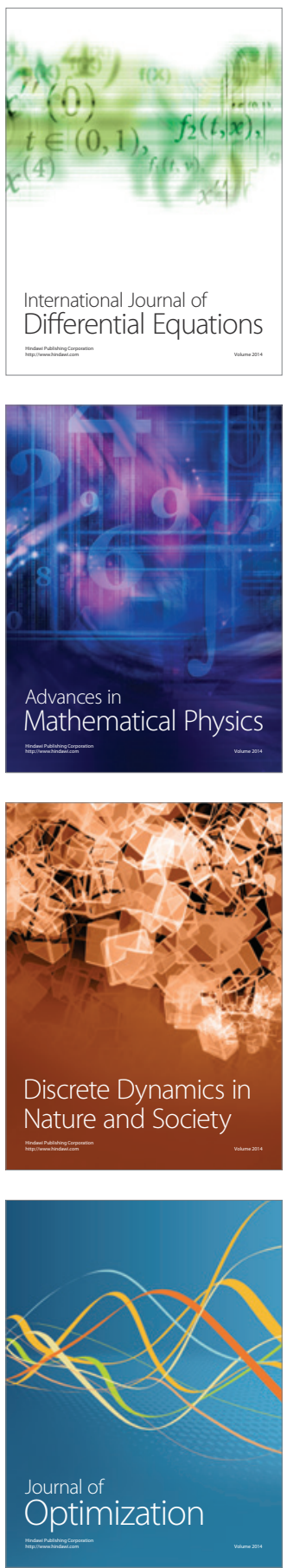